\title{
Efectos del estrés hídrico en crecimiento y desarrollo fisiológico de Gliricidia sepium (Jacq.) Kunth ex Walp.
}

\author{
Effects of water stress on growing and physiological development of Gliricidia sepium \\ (Jacq.) Kunth ex Walp.
}

\author{
Juan Carlos Valverde-Otárola' ${ }^{\odot}$ y Dagoberto-Arias ${ }^{2}$
}

Valverde-Otárola, J.C. y Arias, D. (2020). Efectos del estrés hídrico en crecimiento y desarrollo fisiológico de Gliricidia sepium (Jacq.) Kunth ex Walp. Colombia Forestal, 23(1), 20-34.

Recepción: 16 de abril 2019

\section{Resumen}

El estrés hídrico es una reacción fisiológica de las plantas ante la disponibilidad limitada de agua. Este estudio valoró el efecto del estrés en plántulas de Gliricidia sepium cultivadas en condiciones de invernadero, utilizando plantas testigo y dos tipos de estrés (lineal y cíclico). El estrés generó reducciones en el crecimiento en altura del $30 \%$ y de la lámina foliar del $40 \%$. Las plantas con estrés lineal mostraron a los 98 días un estrés severo con valores fisiológicos mínimos (fotosíntesis $4.51 \mu \mathrm{mol} \mathrm{m}^{-2} . \mathrm{s}^{-1}$, transpiración $6.56 \mu \mathrm{mol} \mathrm{m} \mathrm{m}^{-2} . \mathrm{s}^{-1}$, conductancia 48.6 $\left.\mu \mathrm{mol} \mathrm{m} \mathrm{m}^{-2} \cdot \mathrm{s}^{-1}\right)$; en cambio, las plantas con estrés cíclico si bien se expusieron a un estrés moderado, mostraron recuperación con valores fisiológicos finales estables (fotosíntesis $12.96 \mu \mathrm{mol} \mathrm{m} \mathrm{m}^{-2} \mathrm{~s}^{-1}$, transpiración $6.22 \mu \mathrm{mol} \mathrm{m}{ }^{-2} . \mathrm{s}^{-1}$, conductancia $196.05 \mu \mathrm{mol}$ $\left.\mathrm{m}^{-2} \cdot \mathrm{s}^{-1}\right)$, con un retardo del crecimiento del $30 \%$ con respecto a las plantas testigo, encontrando que en 42 días de estrés la condición es grave.

Palabras clave: plántula, invernadero, fotosíntesis, transpiración, turgencia foliar.
Aprobación: 16 de diciembre 2020

\begin{abstract}
Water stress is a physiological reaction of plants to the limited availability of water. The study assessed the effect of stress on seedlings of Gliricidia sepium grown in greenhouse conditions, using control plants and two types of stress (linear and cyclic). Stress generated reductions in height growth of $30 \%$ and foliar leaf $40 \%$. Plants with linear stress showed severe stress with minimal physiological values at 98 days (photosynthesis $4.51 \mu \mathrm{mol} \mathrm{m}{ }^{-2} . \mathrm{s}^{-1}$, transpiration 6.56 $\mu \mathrm{mol} \mathrm{m}{ }^{-2} . \mathrm{s}^{-1}$, conductance $\left.48.6 \mu \mathrm{mol} \mathrm{m} \mathrm{m}^{-2} . \mathrm{s}^{-1}\right)$, on the other hand, plants with cyclic stress, although they were exposed to moderate stress, showed recovery with stable final physiological values (photosynthesis $12.96 \mu \mathrm{mo} \mathrm{m}^{-2} \cdot \mathrm{s}^{-1}$, perspiration $6.22 \mu \mathrm{mol} \mathrm{m}^{-2} . \mathrm{s}^{-1}$, conductance $196.05 \mu \mathrm{mol} \mathrm{m} \mathrm{m}^{-2} \mathrm{~s}^{-1}$ ), with a $30 \%$ growth delay with respect to the control plants, finding that 42 days of stress the condition is severe.
\end{abstract}

Keywords: seedling, greenhouse, photosynthesis, transpiration, leaf turgor.

1 Laboratorio de Ecofisiología Forestal y Aplicaciones Ecosistémicas (Ecoplant), Escuela de Ingeniería Forestal, Instituto Tecnológico de Costa Rica. Cartago, Costa Rica. Autor para correspondencia jcvalverde@tec.ac.cr

2 Laboratorio de Ecofisiología Forestal y Aplicaciones Ecosistémicas (Ecoplant), Escuela de Ingeniería Forestal, Instituto Tecnológico de Costa Rica. Cartago, Costa Rica. darias@tec.ac.cr 


\section{INTRODUCCIÓN}

El estrés hídrico es una respuesta fisiológica de las plantas a la disminución del agua disponible en el ambiente, lo que incide en un desequilibrio entre la transpiración y la absorción de agua (Myers, 1988; Hammani et al., 2013; Girón et al., 2015). Este fenómeno no solo ocurre cuando hay disponibilidad limitada del agua, sino también por temperaturas en el suelo extremadamente altas o bajas, altos valores de salinidad, baja presión atmosférica o una combinación de los factores mencionados (Girón et al., 2015; Drechsler et al., 2019).

La respuesta de la planta ante la condición de estrés por la falta de agua es inmediata (Roussos et al., 2010; Varone et al., 2012; Tong et al., 2019). El crecimiento se ve afectado debido a la pérdida de turgencia que incide en la reducción de volumen celular y aumento de solutos que generan daños mecánicos celulares que pueden incidir en la reducción de crecimiento, fenómeno que es explicado por el modelo Lockhar-Passioura:

$$
C=m \cdot\left(\psi_{p} \cdot \gamma\right)
$$

Donde $C$ es tasa de crecimiento, $m$ es la extensibilidad pared celular, $\Psi_{p}$ es tensión umbral de la pared celular y $y$ es el umbral de turgencia. Cuando una planta entra en estrés hídrico se da un descenso del valor $m$ y un aumento de $\gamma$, lo que inhibe el desarrollo foliar y el crecimiento del meristemo apical y los meristemos laterales. Maallath et al. (2010) y di Vaio et al. (2013) mencionan que la planta puede desarrollar tres niveles de estrés: 1) mínimo: genera pérdida de turgencia celular, reducción de la tasa de expansión celular, disminución de la síntesis de pared celular y limitaciones en la síntesis de proteínas; 2) moderado: incide en aumentos del ácido abcísico (ABA) y cierre estomático parcial o total; y 3) máximo: en el que la planta produce cavitación de los elementos del xilema, caída de la hoja, acumulación de solutos orgánicos, Ilegando al punto de marchitez de la planta.

Blum (2011) menciona que el estrés hídrico en especies tropicales incide en una reducción significativa de la conductividad estomática y transpiración debido al cierre de estomas; mientras la asimilación fotosintética es afectada conforme el estrés hídrico sea mayor debido a la resistencia de los cloroplastos que se afectan de manera directa conforme la reducción de la expansión foliar aumente y se genera un cierre total de los estomas (Varone et al., 2012). Estudios previos de Otruño et al. (2006) en árboles maduros de Citrus limon y C. aurantium encontraron cambios en la conductividad estomática y turgencia foliar después de 15 días sin irrigación y embolismo en el xilema a los 50 días de iniciado el experimento. Por su parte, Caplan et al. (2019), con 10 especies arbóreas de clima templado de dos a cinco años de edad expuestas a estrés severo, encontraron reducciones en el contenido de humedad, densidad estomática y espesor de hoja en plantas con más de 90 días sin agua, además de reducciones de un $70 \%$ de la asimilación fotosintética y $55 \%$ en la conductividad estomática, encontrando el punto de marchitez permanente entre los 90 a 113 días.

En cuanto especies arbóreas forestales se destaca el estudio de Krause et al. (2012) con tres especies tropicales, allí se encontraron reducciones del $20 \%$ en el crecimiento de los árboles con estrés hídrico, pérdida de un $25 \%$ en la biomasa foliar y reducciones en la tasa fotosintética en un $15 \%$. Ehrenberger et al. (2012) determinaron para Quercus robur el valor de turgencia foliar óptima es de $186 \mathrm{kPa}$ en el cual los niveles fotosintéticos son máximos y tiene mayor actividad fisiológica; a su vez mostró que la turgencia es el primer indicador de estrés hídrico por salinidad en el suelo encontrado reducciones del $25 \%$ en las curvas de turgencia en suelos alfisoles con salinidad superior al $2 \%$ en Europa central. Finalmente, López-López et al. (2018), con árboles maduros de seis especies tropicales, determinaron reducciones del $20 \%$ en transpiración y $15 \%$ en fotosíntesis producto a reducción de la dosificación mensual del agua en un $44 \%$, encontrando pérdida del $50 \%$ del índice del área foliar.

Ante tal panorama, se hace necesario valorar y determinar los niveles de estrés hídrico que pueden 
soportar las especies arbóreas tropicales que se implementan en reforestación, con el fin de generar prácticas de manejo que permitan aumentar su sobrevivencia, especialmente en sus primeros años de establecimiento (Blum, 2011) y mejorar la capacidad de adaptación al cambio climático (Lim, 2013). En el caso de Gliricidia sepium, se trata de una especie tropical de madera preciosa que es muy utilizada en sistemas agroforestales, dado que desarrolla simbiosis con bacterias que contribuye a la nitrificación de suelos, aspecto que mejora la calidad nutricional del suelo. Por su capacidad de rebrote, esta especie presenta características óptimas para su uso en bioenergía debido a su alto poder calórico (Zaharah et al., 1999; Lim, 2013).

El objetivo del presente estudio fue evaluar los efectos del estrés hídrico en el desarrollo fisiológico de plántulas de G. sepium en condiciones de invernadero. El entendimiento de la respuesta al estrés hídrico ayudará a optimizar los aspectos del cultivo forestal con esta especie.

\section{MATERIALES Y MÉTODOS}

\section{Especie y condiciones de estudio}

Se seleccionaron 112 plántulas de Gliricidia sepium de tres meses de edad, procedentes de un huerto semillero del Centro Agronómico Tropical de Investigación y Enseñanza (Catie), ubicado en Turrialba, Costa Rica. Las plántulas seleccionadas se caracterizaron por tener una altura, diámetro a la base y composición foliar similar; posterior a su selección se sembraron en macetas (con dimensiones de $30 \mathrm{~cm}$ de diámetro y $35 \mathrm{~cm}$ de profundidad), en grupos de 4 plantas por maceta. El sustrato utilizado es de fertilidad media y se caracterizó por ser arcilloso, con un $\mathrm{pH}$ promedio de 4.9, libre de piedras y desechos orgánicos; la razón por la cual se usó este tipo de sustrato es debido a dos razones: 1) un suelo con propiedades químicas y físicas homogéneas e ideales para el establecimiento y crecimiento la especie; 2) el suelo al tener una mayor capacidad de retención especialmente por microporos, permite a la planta poder explotar la capacidad máxima de extracción de agua por la raíz conociendo con ello el comportamiento de estrés con un suelo óptimo de establecimiento.

El sitio de estudio se localizó en el vivero de la Escuela de Ingeniería Forestal del Tecnológico de Costa Rica, ubicado en Cartago, Costa Rica $\left(9^{\circ} 50^{\prime} 57.91^{\prime \prime} \mathrm{N} ; 83^{\circ} 54^{\prime} 37.27^{\prime \prime} \mathrm{W}\right)$, situado a una altitud de $1392 \mathrm{~m}$, con una temperatura media anual de $24^{\circ} \mathrm{C}$ y una precipitación anual de 2100 $\mathrm{mm}$, distribuida en su mayoría en 7 meses lluviosos (Instituto Meteorológico Nacional, 2018). El invernadero fue protegido con una capa plástica de poliuretano transparente y malla de sombra de Pead (polímero de alta densidad al 60 \%) de color verde. El sitio presentó una temperatura interna que varió entre $\operatorname{los} 28$ y $32^{\circ} \mathrm{C}$, con una humedad relativa que varió del 80 al $90 \%$, de igual modo las plantas se ubicaron dentro del invernadero por tres semanas antes del inicio de experimento para asegurar una aclimatación adecuada de los individuos al sitio y se les dosificó $50 \mathrm{ml}$ de agua/planta cada dos días. También se controló que recibieran la misma cantidad de horas luz (en promedio 8 horas). La homogeneidad en luminosidad fue verificada con un luxómetro marca Dr. Meter ${ }^{\circledR}$ modelo LX1330B (con valores promedio de 132000 lux). Adicionalmente, para evitar efectos de borde, se colocó una línea de macetas con Gmelina arborea alrededor de las macetas de estudio (con una separación de $15 \mathrm{~cm}$ ).

\section{Modelos de estrés hídrico implementados}

Se desarrollaron tres modelos de estrés hídrico (figura 1) generados a partir de la metodología de Zimmermann et al. (2008) y datos climáticos de la Zona Pacífica Norte de Costa Rica (IMN, 2018). Los modelos de simulación fueron: un testigo (ningún estrés hídrico), estrés hídrico lineal y estrés hídrico cíclico. El estudio simuló los tres modelos por un periodo de 98 días. 
A las plantas del tratamiento testigo se les aplicó de manera constante $300 \mathrm{ml} / \mathrm{planta} /$ semana durante todo el estudio (figura 1). Con el estrés lineal se redujo semanalmente el agua a razón de $50 \mathrm{ml} /$ planta/semana hasta el día 35, posterior al cual se mantuvo la dosis de $50 \mathrm{ml} / \mathrm{semana} /$ planta hasta el día 77 y para las tres últimas semanas del experimento no se aplicó agua a las plantas. En caso de estrés cíclico, se aplicaron las mismas dosis de estrés lineal hasta el día 49, posterior a dicha fecha nuevamente se aplicó un aumento de dosis a razón de $50 \mathrm{ml}$ por planta/semana hasta el día 77, el cual la dosis Ilegó a ser de 300 ml/planta/semana que se mantuvo hasta el día 98.

Para cada simulación de estrés hídrico se utilizaron 8 macetas (32 plántulas). Se utilizó agua a temperatura ambiente y su aplicación fue manual, controlada con una probeta graduada de $500 \mathrm{ml}$.

\section{Evaluación morfométrica de la planta}

Se analizó el crecimiento de las plántulas bajo los distintos tratamientos de estrés, además de los aspectos morfométricos. El crecimiento se enfocó en dos variables: diámetro a la base (medido en la base de la plántula, inicio de la plántula desde el sustrato, que se evaluó con un vernier Minolta graduado en milímetros) y altura total (evaluada desde la base de la planta al meristemo apical, evaluado mediante una cinta métrica graduada en centímetros); ambas variables se midieron al inicio y final del experimento (día 0 y 98) a las mismas plántulas.

Con respecto la biomasa se evaluó tanto al día 0 como 98 (debido a la homogeneidad del material al inicio de experimento se cosecharon plántulas excedentes para la obtención de dichos valores), la biomasa seca se obtuvo con la extracción completa de la planta la cual se seccionó en: hojas, ramas, tallo y raíz, cada parte se secó por 72 horas a una temperatura de $105^{\circ} \mathrm{C}$ y se pesó con una Balanza Ocony en gramos. Con respecto al número de hojas, se contabilizaron de manera manual en cada planta y el área foliar específica (AFE), se determinó a partir de la división de área de la lámina foliar (en $\mathrm{cm}^{2}$ ) entre el peso de la lámina en seco (en gramos).

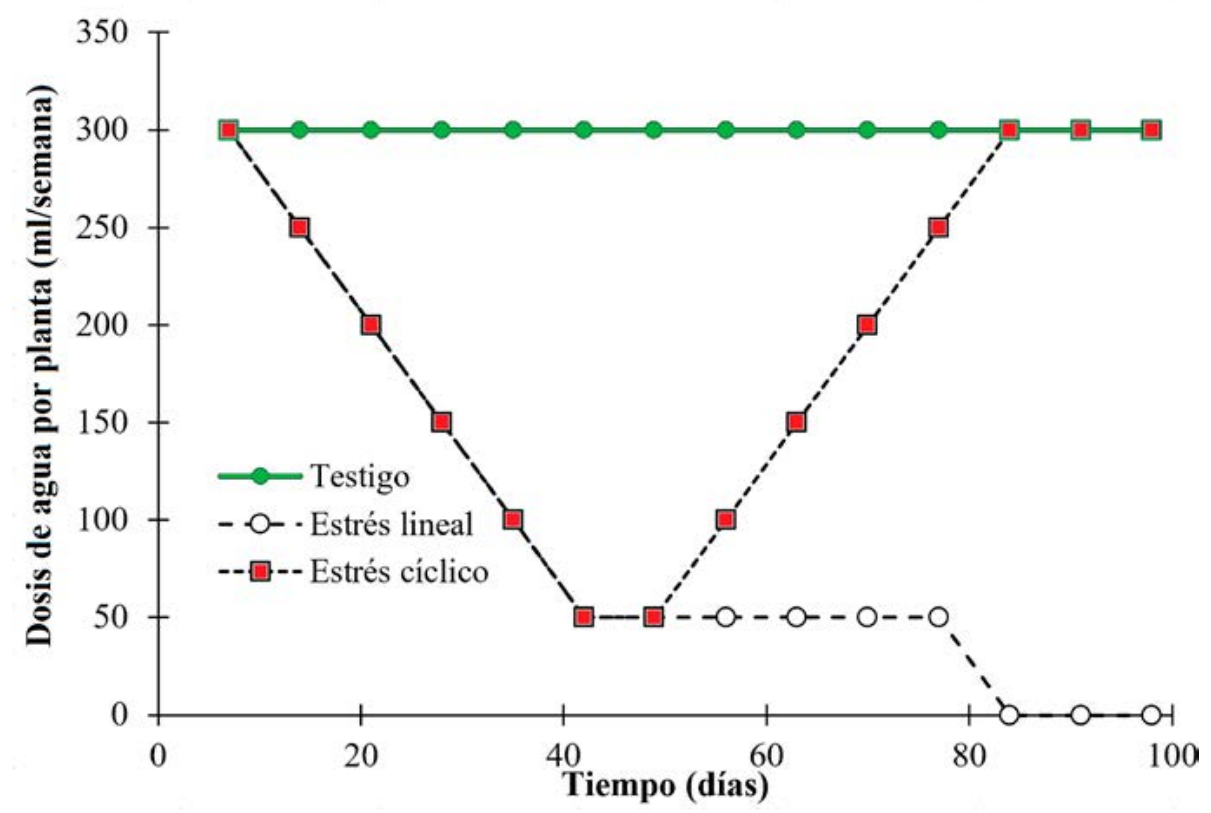

Figura 1. Dosificación de agua por planta semanal aplicada a las plantas de G. sepium bajo tres tratamientos de manejo hídrico en condiciones de invernadero. 


\section{Parámetros fisiológicos evaluados}

A cada planta de G. sepium se le seleccionó una hoja compuesta de edad intermedia, libre de defectos para valorar los siguientes parámetros: tasa fotosintética neta $(A n)$, transpiración $(E)$, área foliar específica (AFE), contenido relativo de clorofila (medido con SPAD), conductividad estomática (Gs), contenido de humedad foliar (CHF) y turgencia foliar (Pp). Las variables An, E y AFE se evaluaron al inicio (día 0) y el día final de la prueba (día 98), mientras que la turgencia foliar se evaluó durante todo el experimento, programando el equipo para captación de datos cada 15 minutos. Las variables restantes se valoraron cada 14 días. Las mediciones se realizaron entre las 09:00 y 11:00 (GMT-6 Central América), Ilevando control de la temperatura, humedad relativa y luminosidad con el fin que su variabilidad no infiriera en los datos obtenidos. A continuación, se especifica el procedimiento realizado para cada parámetro:

1. Tasa fotosintética neta y transpiración: ambos parámetros se evaluaron con un medidor de intercambio gaseoso CIRAS-II (PP Systems ${ }^{\circledR}$, EE. UU.). El CIRAS-II se calibró con un flujo constante de $\mathrm{CO}_{2}$ de 380 ppm y se programó para realizar las curvas de asimilación fotosintética de 0 a $2000 \mu \mathrm{mol}$ (quanta) $\mathrm{m}^{-2} \cdot \mathrm{s}^{-1}$, en intervalos de 200 $\mu \mathrm{mol}$ (quanta) $\mathrm{m}^{-2} \cdot \mathrm{s}^{-1}$, en un área estándar de 2.5 $\mathrm{cm}^{2}$ de la hoja. La cubeta de medición del instrumento se ubicó en el lado derecho de todas las hojas para estandarizar el proceso de medición.

2. Valor del contenido relativo de clorofila en las hojas: las mediciones del contenido potencial de clorofila se realizaron con un SPAD-502 (Konica Minolta $($ ) que cuenta con un área efectiva de valoración de $5 \mathrm{~mm}^{2}$. Dado que la especie tiene hojas compuestas, se tomó la medición a cada hojuela que la conformaba; únicamente se omitieron las hojuelas que no se adaptaran al punto de entrada del equipo o que mostraran algún defecto.

3. Conductividad estomática: se evaluó mediante el uso de un Leaf Porometer CS-1 (Degacon
Devices, Inc $\mathbb{R})$, el cual cuenta con una cámara de apertura de medición de $6.35 \mathrm{~mm}$ de diámetro; el equipo previamente se comparó con los datos de conductividad estomática del Ciras II para evitar variaciones entre sí; su implementación se debió a su rapidez de medición, lo que permitió tomar tres mediciones por planta en la primera hojuela de la hoja compuesta.

4. Turgencia foliar: este parámetro se determinó mediante unidades ZIM-probe (Yara®) de 82 $\mathrm{mm}^{2}$ de cobertura, programada para tomar los datos cada 15 minutos según recomienda la metodología de Zimmermann et al. (2008); los valores de turgencia foliar (Pp) son generados a partir del principio de la ecuación 1.

$$
P_{p}=\left(\frac{b}{a P_{c}+b}\right)^{\frac{1}{a}} \cdot F_{a} \cdot P_{\text {clamp }}
$$

Donde $a$ y $b$ son constantes, $F_{a}$ es el factor de atenuación específico de la hoja y $\mathrm{P}_{\text {clamp }}$ es un factor de atenuación del sensor. Las hojas seleccionadas para adquirir datos con el Zim-probe, se caracterizaron por no tener presencia de agua superficial ni defectos o decoloraciones atípicas que generan distorsiones en la medición.

5. Contenido de humedad foliar: de cada planta se extrajo una hoja compuesta que se pesó y secó a una temperatura de $80^{\circ} \mathrm{C}$ por 48 horas para obtener el peso seco que permitió calcular el contenido de humedad con la ecuación 2.

$$
\operatorname{CHF}(\%)=\left(\left(\frac{P S}{P V}\right)-1\right) \cdot 100
$$

Donde CHF es el contenido de humedad en porcentaje, PS es el peso seco de la hoja compuesta (en gramos) y PV es el peso verde de la hoja compuesta (en gramos).

6. Área foliar específica: la totalidad de hojas colectadas de cada planta se escanearon y analizaron en el programa WIN FOLIA versión 2012 pro (Regent Instrument, 2012), poniendo en funcionamiento el sistema de caracterización de 
píxeles para determinar el SLA promedio de cada planta y tratamiento.

7. Análisis de biomasa: en el inicio de la prueba se cosecharon 16 plántulas para calcular el peso seco de hojas, raíz, tallo y total; posteriormente, en el día 98 se desarrolló una cosecha de las restantes 96 plantas, cada una se segmentó en sus distintos componentes y se secaron a $100^{\circ} \mathrm{C}$ por un periodo de 48 horas para calcular el peso seco.

\section{Diseño experimental y análisis de resultados}

Se aplicó un diseño experimental simple aleatorio, en el cual se evaluó si existen diferencias significativas entre los tratamientos de estrés hídrico en cada fecha de medición, para lo cual se puso en marcha un análisis de varianza de una vía (One way Andeva) y a las variables que mostraron diferenciaciones se les aplicó prueba de Tukey con una significancia de 0.05. Los análisis se realizaron con en el programa Statistica 9.0 (Statsoft, 2015). En cada análisis se verificaron los supuestos de normalidad de los residuales y la homogenización de las varianzas.

\section{RESULTADOS}

\section{Crecimiento y desarrollo de las plantas}

En la tabla 1 se presentan los valores de crecimiento, biomasa seca y caracterización de las hojas de los individuos en el día de inicio y día final del experimento; es importante destacar que en el día 0 los parámetros analizados no mostraron diferencias entre sí. El diámetro a base promedio en día 0 fue de $4.03 \mathrm{~mm}$, posterior del ensayo su valor aumentó a $5.83 \mathrm{~mm}$, sin mostrar diferencias significativas entre los tratamientos, por su parte la altura inicialmente fue de $257.2 \mathrm{~mm}$, y varió significativamente en el día 98 obteniendo que las plantas testigo mostraron las alturas mayores (398.8 $\mathrm{mm}$ ) en comparación a las plantas con estrés (de $291.9 \mathrm{~mm}$ ) que no presentaron diferenciación de altura entre los dos tratamientos.

Con respecto a la biomasa seca, en día 0 en promedio era $1.154 \mathrm{~g}$, correspondientes a $5 \%$ en hojas, 72 \% en tallo y 23 \% raíz, el comportamiento varió en día 98 mostrando en las plantas testigo un aumento del $2.01 \mathrm{~g}$ dispuestos en el tallo y raíz principalmente. En cambio, las plantas en estrés cíclico con un aumento de $1.586 \mathrm{~g}$ acumulado en fuste y el estrés lineal con el menor aumento de tan solo $0.82 \mathrm{~g}$, apenas perceptible en tallo y raíz.

En cuanto al análisis de la hoja en día 0, las plantas presentaron una cantidad media de 6.5 hojas con un AFE de $13.6 \mathrm{~cm}^{2} . \mathrm{g}^{-1}$; en cambio, en el día 98 las plantas testigo duplicaron la cantidad de hojas en la planta con 12.9 con un AFE mayor de $22.5 \mathrm{~cm}^{2} . \mathrm{g}^{-1}$, mientras las plantas con estrés lineal obtuvieron disminución de hojas presentando en promedio 2.0/planta, con un aumento mínimo de AFE a $8.4 \mathrm{~cm}^{2} \cdot \mathrm{g}^{-1}$. Finalmente, las plantas con estrés cíclico presentaron 5.9 hojas.planta ${ }^{-1}$ con un aumento del AFE a $15.6 \mathrm{~cm}^{2} . \mathrm{g}^{-1}$.

\section{Asimilación fotosintética neta y transpiración}

Con respecto a la asimilación fotosintética neta (An), en las plantas en día 0 no mostraron variaciones entre tratamientos (figura 2), lo que permite ver un aumento de An exponencial en función al FFDP, con una An max de (14.95 \pm 2.16$) \mu \mathrm{mol}$ $\mathrm{m}^{-2} \cdot \mathrm{s}^{-1}$. En el día 98 la An varió con cada tratamiento, si bien mantuvo la tendencia de aumento del An en función de PPFD. Las plantas testigo presentaron una curva de asimilación superior a las plantas en estrés, obteniendo un An max de $(25.6 \pm 1.33) \mu \mathrm{mol} \mathrm{m} \mathrm{m}^{-2} \cdot \mathrm{s}^{-1}$, caso distinto a las plantas expuestas en estrés que entre sí mostraron diferenciaciones en sus curvas de asimilación después de los $750 \mu \mathrm{mol} \mathrm{m} \mathrm{m}^{-2} \cdot \mathrm{s}^{-1}$. En estrés cíclico se dio una An max de $(12.96 \pm 2.60) \mu \mathrm{mol} \mathrm{m}{ }^{-2} \cdot \mathrm{s}^{-1}$, correspondiente a un $49 \%$ menos con respecto a la An max de las plantas testigo y un $13 \%$ menos que las plantas en día 0. Caso similar se dio con las plantas de estrés lineal que presentaron el An 
Tabla 1. Valores inicial y final de crecimiento, masa seca y característica de la hoja en las plantas testigo y con estrés lineal y cíclico de G. sepium

\begin{tabular}{|c|c|c|c|c|c|c|}
\hline \multirow{3}{*}{ Variable } & \multicolumn{6}{|c|}{ Tratamiento / día } \\
\hline & \multicolumn{3}{|c|}{ Día 0} & \multicolumn{3}{|c|}{ Día 98} \\
\hline & Testigo & Estrés lineal & Estrés cíclico & Testigo & Estrés lineal & Estrés cíclico \\
\hline \multicolumn{7}{|l|}{ Crecimiento } \\
\hline Diámetro en base $(\mathrm{mm})$ & $3.96 .^{\mathrm{a}}(0.36)$ & $4.05 .^{a}(0.39)$ & $4.06 .{ }^{a}(0.26)$ & $6.45 .^{a}(0.80)$ & $5.35 .^{a}(1.61)$ & $5.69 .^{a}(1.09)$ \\
\hline Altura $(\mathrm{cm})$ & $253.1 .^{\mathrm{a}}(20.5)$ & $259.0^{\mathrm{a}}(17.7)$ & $259.5 .^{\mathrm{a}}(15.66)$ & 398.8. ${ }^{\mathrm{a}}(17.1)$ & $284.1^{\mathrm{b}}(41.1)$ & $299.6^{b}(46.6)$ \\
\hline \multicolumn{7}{|l|}{ Peso seco (g) } \\
\hline Total & $1.191 .^{\mathrm{a}}(0506)$ & $1.156 .^{\mathrm{a}}(0.479)$ & $1.115 .^{\mathrm{a}}(0.458)$ & $3.199 .^{\mathrm{a}}(0.125)$ & $1.974^{\mathrm{c}}(0.554)$ & $2.740^{\mathrm{b}}(0.206)$ \\
\hline Hojas & $0.066 .^{\mathrm{a}}(0.033)$ & $0.070 .^{\mathrm{a}}(0.040)$ & $0.060 .^{\mathrm{a}}(0.039)$ & $0.125 .^{\mathrm{a}}(0.018)$ & $0.084^{b}(0.031)$ & $0.088^{b}(0.041)$ \\
\hline Ramas & $0.869 .^{\mathrm{a}}(0.156)$ & $0.830 .^{\mathrm{a}}(0.114)$ & $0.799 .^{a}(0.206)$ & $2.061^{\mathrm{a}}(0.130)$ & $1.528^{\mathrm{c}}(0.120)$ & $1.748^{\mathrm{b}}(0.167)$ \\
\hline Raíz & $0.256 .^{\mathrm{a}}(0.189)$ & $0.256 .^{\mathrm{a}}(0.189)$ & $0.256 .^{\mathrm{a}}(0.189)$ & $1.043^{\mathrm{a}}(0.468)$ & $0.363^{\mathrm{c}}(0.159)$ & $0.550^{\mathrm{b}}(0.240)$ \\
\hline \multicolumn{7}{|l|}{ Hojas } \\
\hline Número de hojas & $6.9^{\mathrm{a}}(3.2)$ & $6.1^{\mathrm{a}}(2.9)$ & $6.4^{\mathrm{a}}(2.5)$ & $12.9^{\mathrm{a}}(3.3)$ & $2.0^{c}(2.0)$ & $5.9^{b}(2.8)$ \\
\hline AFE $\left(\mathrm{cm}^{2} \cdot \mathrm{g}^{-1}\right)$ & $12.5(3.9)$ & $14.5(4.1)$ & $13.8(3.8)$ & $22.4^{\mathrm{a}}(4.5)$ & $8.9^{\mathrm{c}}(5.1)$ & $15.5^{\mathrm{b}}(6.5)$ \\
\hline
\end{tabular}

Nota: letras distintas muestran diferencias significativas con $\mathrm{P}<0.05$.

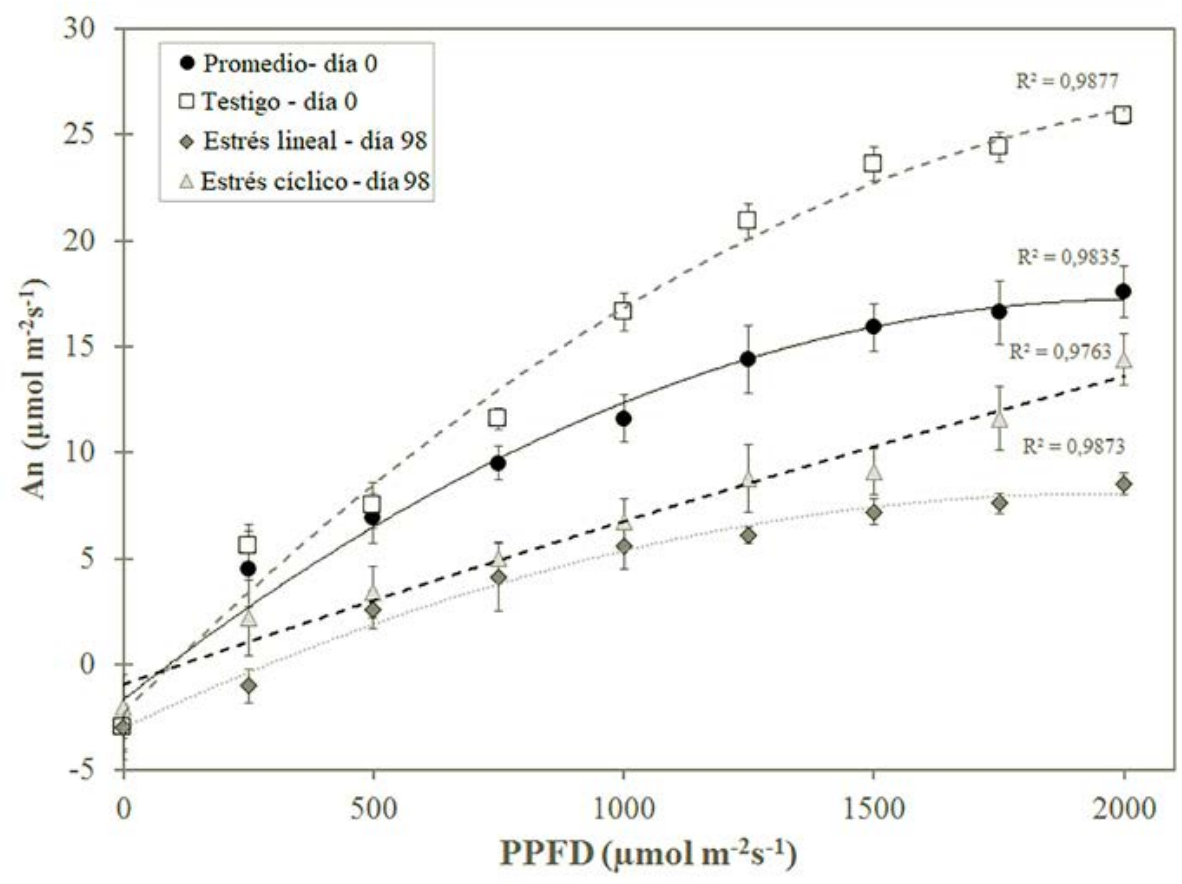

Figura 2. Curvas de Asimilación fotosintética neta (An) con respecto a la Photosynthetic Photon Flux Density (PPFD), obtenida en día 0 y día 98 en plantas de G. sepium bajo tres tratamientos de estrés hídrico en condiciones de invernadero. 
máximo de $(4.51 \pm 1.16) \mu \mathrm{mol} . \mathrm{m}^{-2} . \mathrm{s}^{-1}$, valor representante una reducción de An de un $83 \%$ con respecto al An testigo en día 98 y $69.9 \%$ menos con An max en día 0.

En cambio, con la transpiración (E) en día 0 no se percibieron diferencias significativas entre tratamientos, mostrando un valor medio de $(5.66 \pm 1.82)$ $\mu \mathrm{mol} \mathrm{m} \mathrm{m}^{-2} \cdot \mathrm{s}^{-1}$, que varió considerablemente en el día 98: las plantas testigo mostraron un aumento de $\mathrm{E}$ a $(11.36 \pm 3.44) \mu \mathrm{mol} . \mathrm{m}^{-2} \cdot \mathrm{s}^{-1}$, significativamente mayor a las plantas en estrés que presentaron valores de $(6.56 \pm 2.44) \mu \mathrm{mol} \mathrm{m}{ }^{-2} \cdot \mathrm{s}^{-1}$ en estrés cíclico y $(6.22 \pm 1.99) \mu \mathrm{mol} . \mathrm{m}^{-2} . \mathrm{s}^{-1}$ con estrés lineal.

\section{Conductividad estomática, SPAD y contenido de humedad foliar}

Se encontraron diferencias significativas de la conductividad estomática (Gs) y el contenido de humedad foliar (CHF), mostrando una tendencia similar en su comportamiento (figuras 3 a y $3 b$, respectivamente). Los tratamientos no mostraron diferencias significativas hasta el día 14, posteriormente, las plantas testigo estabilizaron sus valores hasta día 98, obteniendo un Gs promedio de (250.16 \pm 2.33$) \mu \mathrm{mol} \mathrm{m} \mathrm{m}^{-2} \cdot \mathrm{s}^{-1}$ y CHF promedio del $(89.5 \pm 3.95) \%$; en cambio las plantas con estrés presentaron reducciones de Gs y CHF, obteniendo valores similares hasta el día 42 de $97.62 \mu \mathrm{mol}$ $\mathrm{m}^{-2} \cdot \mathrm{s}^{-1}$ (Gs) y $59.45 \%$ (CHF); luego del día 42 los comportamiento de ambos grupos variaron: con el estrés cíclico se presentó la tendencia de aumento de Gs y FMC hasta el día 98 obteniendo un valor medio de $196.05 \mu \mathrm{mol} \mathrm{m}{ }^{-2} . \mathrm{s}^{-1}$ (Gs) y $78.3 \%$ (FMC). En cambio, con estrés lineal la tendencia de disminución de los parámetros se mantuvo y estabilizó después del día 70 con $48.6 \mu \mathrm{mol} . \mathrm{m}^{-2} \cdot \mathrm{s}^{-1}$ (Gs) y $46.3 \%$ (FMC).

Para los valores obtenidos con el SPAD (figura 3c) se presentaron tendencias distintas, hasta el día 28 los tres tratamientos no mostraron diferencias y con una tendencia de aumento gradual del SPAD; a partir del día 42 se dan diferencias y se obtuvo que los valores SPAD de las plantas testigo y con estrés cíclico superaron significativamente a las plantas con estrés lineal, que mostraron una tendencia a reducir su valor hasta el final de la prueba mostrando un aumento de la variabilidad de los valores SPAD conforme avanzó el

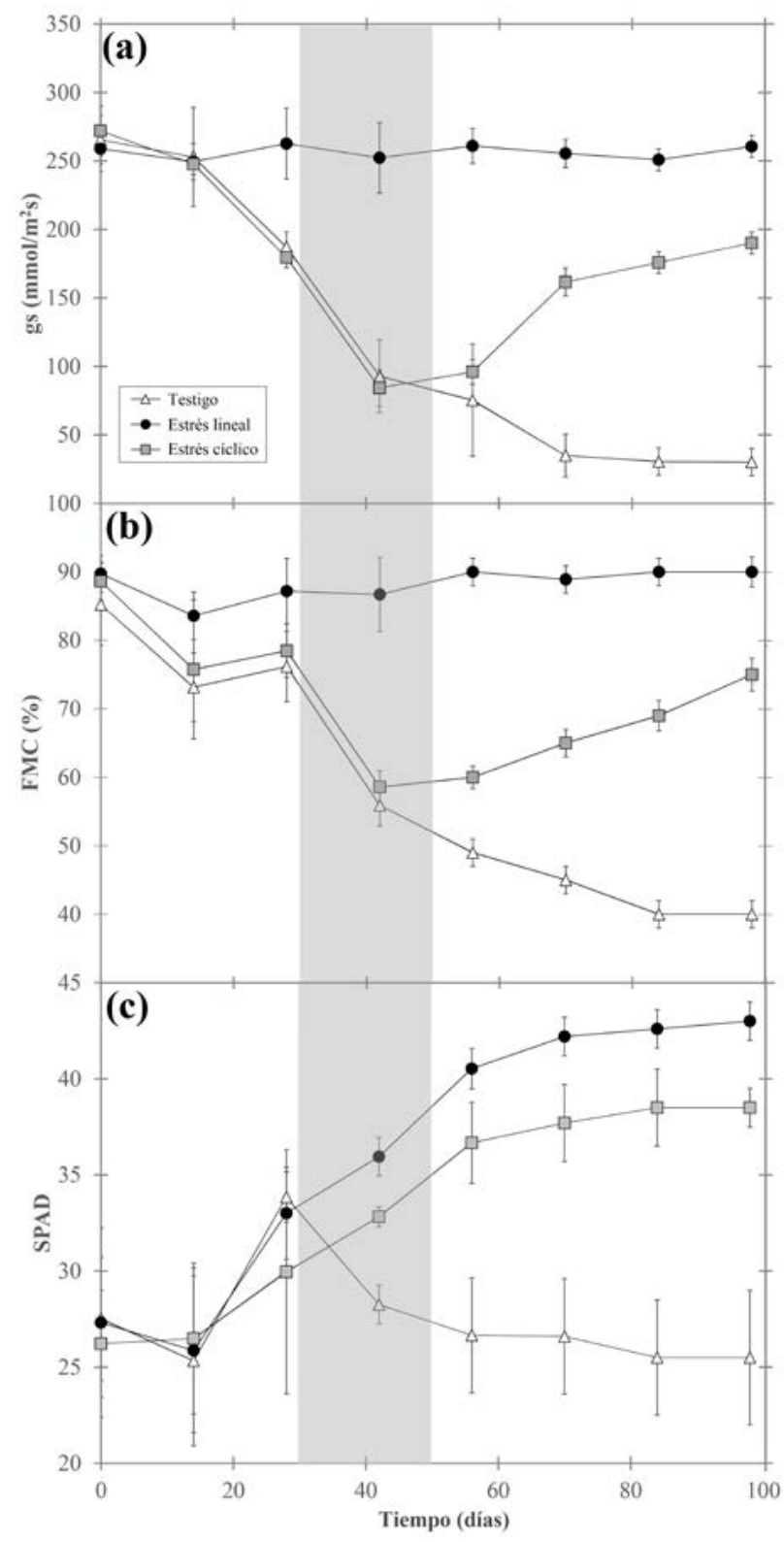

Figura 3. Variabilidad de la conductividad estomática (Gs), contenido de humedad foliar (FMC) y contenido relativo de clorofila (SPAD) en función al tiempo obtenidos en plantas de G. sepium bajo tres tratamientos de estrés hídrico en condiciones de invernadero. 
tiempo. En cambio, las plantas testigo y en estrés cíclico mantuvieron un aumento del SPAD hasta el día 72, momento en el cual se estabilizaron los valores, manteniendo la diferenciación siendo que las plantas testigo presentaron valores mayores a los de estrés cíclico.

\section{Turgencia foliar}

En la figura 4 se muestra la variación de la turgencia foliar (Pp) y la temperatura en función del tiempo. El comportamiento de Pp en los primeros 10 días (figuras $4 a, 4 b$ y 4 c) es similar en los tres tratamientos, los valores mínimos de Pp se presentaron entre $12: 00$ y 06:00 con turgencia de (185 a 199) $\mathrm{kPa}$ a una temperatura mínima de 15 a $18^{\circ} \mathrm{C}$ (figura 4d); este comportamiento varió entre las 06:00 y $11: 30$, con una clara tendencia a aumentar tanto Pp como la temperatura que presentaron los valores máximos entre las 11:30 y 14:00 horas con turgencia de (240 a 260) kPa a temperaturas de (43 a 46) ${ }^{\circ} \mathrm{C}$, posterior de las 14:00 horas nuevamente se dio la tendencia de reducción de Pp hasta las 12:00, momento en el cual vuelve a iniciarse el ciclo de variación.

En el periodo de los días 40 al 49 únicamente las plantas testigo (figura 4e) mantuvieron el comportamiento de turgencia inicial, pero con un aumento de Pp mínimo de (195 a 205) kPa y máximo de (250 a 260) kPa. Mientras que las plantas con estrés (que en ese momento recibían una dosis semanal de $50 \mathrm{ml} /$ planta) mostraron oscilaciones significativas entre el día y la noche (figuras 4 e y 4f). La Pp mínima empezó a aumentar en la noche de 190 kPa (día 140) a 210 kPa (día 49), mientras que durante el día se dieron disminuciones del valor máximo, siendo para el día 40 un valor de 250 kPa y de 165 kPa para el día 49, ocurriendo que en los días 46, 47 y 48 no se diera un comportamiento predecible de la turgencia, sino que presentara oscilaciones considerables que incidieran que en el día 47 se mostrara una nueva tendencia en la cual los Pp máximos se obtuvieran en horas de la noche y Pp mínimos en el día.
Finalmente, entre los días 89 y 98 se repite el comportamiento de la planta testigo, pero con aumentos en la Pp durante la noche que varió de 205 a 215 kPa; mientras que, durante el día, la turgencia disminuyó a 220 a $230 \mathrm{kPa}$ (figura 4i). Las plantas con estrés hídrico recuperaron turgencia en comparación al día 49 (con la dosis máxima de agua semanal de $300 \mathrm{ml} /$ planta), mostrando valores máximos de 250 a $260 \mathrm{kPa}$ entre las 11:30 y 14:00, en la noche presentaron turgencia mínima de 180 a 190 kPa. Finalmente, las plantas con estrés lineal (en ese momento no tenía dosis alguna de agua) mantuvo el comportamiento inverso de turgencia obtenido después del día 48, presentando el Pp máximo de 150 a 160 kPa en horas de la noche (figura 4k). En cambio, el Pp mínimo se dio en el día con una variación de 100 a 110 kPa.

En todo momento del experimento (figuras $4 d$, 4 h y 4 l) la temperatura mostró leves variaciones, indicando temperaturas mínimas en la noche de 15 a $20^{\circ} \mathrm{C}$ y máximas en el día de 44 a $47^{\circ} \mathrm{C}$.

\section{DISCUSIÓN}

Una reducción en el crecimiento de las plantas, en conjunto con el estancamiento en la acumulación de masa seca, así como una disminución del AFE y caída de hojas (tabla 1) resultaron ser las evidencias visuales del estrés hídrico en las plantas de $G$. sepium, lo cual es congruente con los resultados que mencionan Pedrero et al. (2014) en plantas tropicales. Es importante destacar que las plantas por procesos evolutivos han desarrollado mecanismos de tolerancia y evitación al estrés hídrico. Los mecanismos de tolerancia son adaptaciones fisiológicas que permiten a la planta acumular agua o dosificarla con el fin de sobrevivir periodos de sequía, mientras los mecanismos de evitación son medidas de respuesta a las condiciones de estrés extendidas y que se tiende a modificar según el sitio. Maallath et al. (2010), Sánchez-Costa, Poyatos y Sabate (2015) y Szota et al. (2019) identificaron cuatro mecanismos de evitación al estrés en 

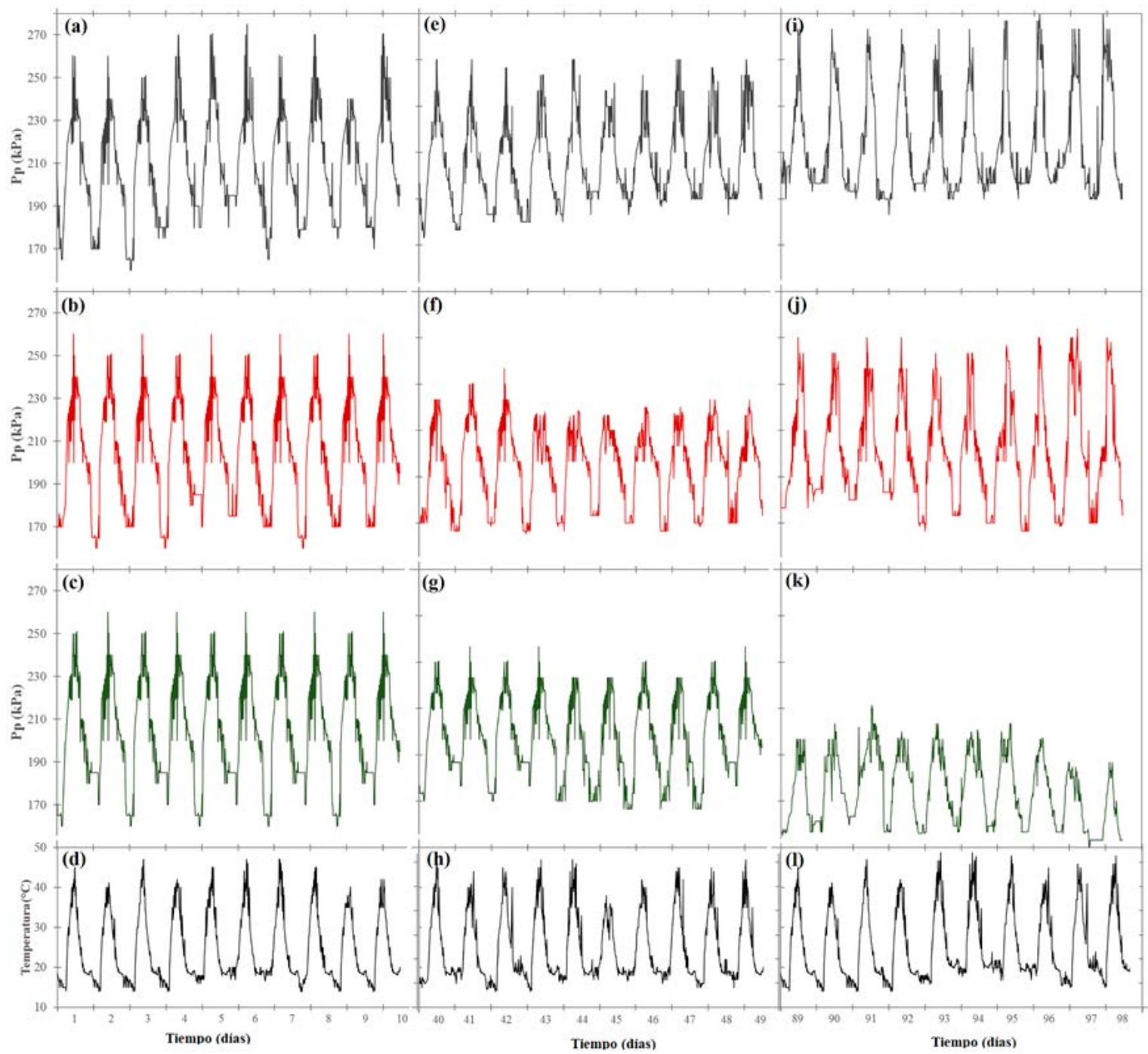

Figura 4. Turgencia foliar (Pp), temperatura y humedad relativa en los períodos del día 0 al 10, día 40 al 49 y el día 89 al 94, en plantas de G. sepium bajo tres tratamientos de estrés hídrico en condiciones de invernadero.

especies tropicales: 1) variando la superficie foliar por la que el agua se transpira, 2) controlando la pérdida de agua por unidad de superficie foliar mediante los estomas, 3) modificando la conductividad hidráulica entre las distintas partes de la planta para minimizar el embolismo y 4) adaptando el sistema radicular para mejorar la captación de agua.

En el presente estudio se verificaron reducciones superiores al $20 \%$ del AFE (tabla 1), con disminuciones del $50 \%$ de la cobertura de hojas y reducciones superiores al $45 \%$ en la transpiración de las plantas en estrés, esto se puede explicar en función de las conclusiones de Zimmermann, Westhoff y Zimmermann (2007). Westhoff et al. (2009) y Camareroa et al. (2019) mencionan que el estrés de una planta está altamente ligado a la turgencia, debido a que la disponibilidad de agua que tenga la planta afecta su desarrollo fisiológico. Guerfel et al. (2009) encontraron para variedades de olivo un efecto directo de la pérdida de turgencia en el desarrollo de las plantas, definiendo qué pérdidas de turgencia de un 10 al $15 \%$ podría 
retardar el crecimiento de los árboles juveniles en un $30 \%$ debido al impacto que se genera en la producción de proteínas y acumulación de solutos orgánicos, que pueden generar intoxicaciones y daños foliares significativos. En el presente estudio se encontró que en el día 98 se dio un comportamiento diferente entre las plantas control y las de estrés lineal que, según Blum (2011), se explica por un estrés critico que podría incidir en el marchitamiento de las plantas.

El proceso de estrés hídrico obtenido se pudo categorizar en las fases formuladas por Maallath et al. (2010), di Vaio et al. (2013), de la Rosa et al. (2014) y Sánchez-Costa et al. (2015) que realizan la clasificación en estrés mínimo, moderado y severo. Utilizando este criterio se encontraron diferencias significativas en Gs, LMC y Pp (este último facilitó identificar cada fase). La primera fase de estrés mínimo es atribuida por Maallath et al. (2010) a cambios metabólicos y físicos significativos, se da una disminución en la síntesis de proteínas que incide en la reducción del espesor de las paredes celulares; además, un aumento de transpiración, reducción de turgencia foliar y de la expansión foliar. También se incide en cierres estomáticos de tipo hidropasivos de manera esporádica en los momentos de mayor temperatura en el día (Blum, 2011); este comportamiento se identificó después del día 12 cuando se evidenciaron disminuciones de un $20 \%$ en CHF, leves reducciones de Gs (inferiores a $22 \mu \mathrm{mol} . \mathrm{m}^{-2} . \mathrm{s}^{-1}$ ) y cambios visuales en la angulación foliar. En el caso de G. sepium se trata de una especie cuyas hojas presentan peciolos gruesos que permiten generar movimientos de la hoja para reducir o aumentar la incidencia de la lámina foliar al calor (Fernández et al. 2011), esto producto de la pérdida de Pp que varió 10 a 15 kPa similares a los presentados por Rodríguez-Domiguéz et al. (2012) con arbustos juveniles de olivo.

La segunda fase de estrés considerada como moderada (figuras 3 y 4) se presentó a partir del día 42. Los valores de Pp mostraron variaciones superiores a los $60 \mathrm{kPa}$ y con un comportamiento atípico al visto en la fase anterior en la que se obtuvo aumentos del Pp durante el día (llegando a valores máximos a las 12:00) y, posterior a ello, la presión disminuyó alcanzando valores mínimos en la noche debido al movimiento de agua foliar. Zimmermann et al. (2004) y Ehrenberger et al. (2012) determinaron que en el día la fotosíntesis, conductividad estomática y la transpiración es alta, lo que provoca que la planta deba movilizar agua del xilema y raíces para nivelar la presión en las hojas (Zimmermann et al., 2007), si este movimiento es inferior a $50 \mathrm{kpa}$ se puede asumir que la planta tiene capacidad suficiente de respuesta. Sin embargo, en el nivel moderado la poca disponibilidad de agua en el ambiente (figura 4) incide en un comportamiento poco uniforme de Pp debido a la capacidad de respuesta que a su vez es limitada por parte de la planta que puede incidir en que se produzca un comportamiento inverso del $\mathrm{Pp}$, puesto que la planta no tiene la capacidad de responder el desequilibrio de agua, lo que incide en el cierre de estomas (que se presentó con reducciones de Gs en día 42), pérdidas de hasta un $50 \%$ del contenido de humedad foliar y aumento de la producción de ABA. Esta última medida con el fin de generar regulaciones en las hojas y evitar que la cantidad de agua en las láminas foliares aumente por la dificultad de los estomas en cerrarse ante la falta de agua. Blum (2011) destaca que en esta etapa se inicia la caída de hojas viejas, según menciona por ser las hojas con menor capacidad de control estomático, y ser la primera en que la fotosíntesis disminuye en condición de estrés. Es importante destacar que aún en esta fase la planta se puede recuperar, pero con retardos en crecimiento. Rodríguez-Domiguéz et al. (2012) reportaron una disminución en el desarrollo de un 20 a 35 \% en plantas jóvenes de olivo en condiciones de estrés moderado.

Finalmente, en la fase 3 (que se presentó a partir del día 70), considerada de no retorno en la cual la planta tiene la conductividad estomática y CHF mínimos (figuras $3 a$ y $3 b$ ), con disminuciones superiores al $60 \%$ de los valores iniciales, la turgencia tiene diferenciaciones superiores a $60 \mathrm{kPa}$ y 
la mayoría de las hojas han caído y disminuido de tamaño, esto con el fin de reducir el área de pérdida de agua, limitar la fotosíntesis en especial la generación de ATP por el consumo energético que genera (Blum 2011). Además, se genera cavitación y embolismo en el sistema radicular, lo que inhibe la funcionalidad del xilema y la capacidad de absorción de agua desde el suelo. La cantidad de solutos orgánicos presentes en las hojas, al no poder ser disuelto y transferidos de las hojas, aceleran la marchitez foliar debido a los cambios de $\mathrm{pH}$ que generan y las afectaciones en el cierre de estomas los cuales, si bien en su mayoría se encuentran cerrados, la planta no tiene la capacidad suficiente de controlar.

Con evidencia experimental en este estudio las plantas con estrés lineal en el día 98 no tienen la capacidad de recuperación. Las plantas sometidas a un estrés cíclico mostraron recuperación sustancial, pero con retrasos en su desarrollo. Esta recuperación fue posible por los bajos efectos del embolismo, ya que Zimmermann et al. (2004), Zimmermann et al. (2007) y Rodríguez-Domiguéz et al. (2012) mencionan que esta condición inhibe la capacidad de recuperación de la planta, pues las bolsas de oxígeno evitan que den los procesos de presión necesarios en la planta causando su muerte o en el mejor de los casos que pueda rebrotar en las áreas libres de embolismo.

Con la información generada se puede tener un mayor entendimiento del manejo silvicultural de la especie con potencial uso en programas plantaciones con fines múltiples como el energético. Zimmermann et al. (2007) destacan que conforme sea mayor el conocimiento hidráulico y resistencia fisiológica a exposiciones reducidas de agua se puede determinar el grado de tolerancia de la especie y adelantar el proceso de siembra antes del inicio de la época lluviosa con el fin aumentar los rendimientos de reforestación. Por su parte, Blum (2011) menciona que conocer el punto de marchitez permanente permite definir estrategias de protección de cultivos ante épocas secas extensas. En este caso, al conocer que posterior de 42 días con déficit hídrico la planta puede morir, se pueden planificar estrategias de retención de humedad en el sitio y con ello se garantiza su sobrevivencia de los individuos. Además, Pedrero et al. (2014) recomiendan que para manejo de las especies que se conozca su punto de marchitez permanente se debe disponer de medidas de reducción de estrés (administración artificial de agua), prevenir con uso de retenedores de agua en el momento de siembra (hidrogeles), estudios detallados de las condiciones climáticas de la región (conocimiento de la duración y periodo del año en que se presenta la época seca), con ello se puede reducir el riesgo de mortalidad o pérdida de áreas reforestas.

\section{CONCLUSIONES}

Se determinó que el estrés hídrico incide significativamente en el crecimiento y desarrollo fisiológico del G. sepium. En cuanto al crecimiento, este se reduce en un $30 \%$ con énfasis en la altura y biomasa que son significativas con el estrés lineal; mismo comportamiento se presentó con todas las variables fisiológicas (An, SPAD, Gs, CHF y Pp), ya que las plantas se encontraron en punto de estrés moderado a los 42 días, posteriores a los cuales la planta no tiene capacidad alguna de recuperar y entra en estrés crítico, punto en el cual se dio pérdida foliar, incapacidad de recuperación de la turgencia y, fisiológicamente, una reducción hasta del $40 \%$ de las funciones.

Por tanto, luego de 42 días las plantas no tienen posibilidad de recuperarse y entran a un estrés crítico que incide en el punto de marchitez permanente, por lo que si se da una intervención la recuperación es factible, con reducción en el crecimiento y capacidades fisiologías, pero con posibilidad de que la especie pueda continuar sobreviviendo, tal como se mostró en el estrés cíclico. Se comprendió que la especie Gliricida sepium tiene buena capacidad para tolerar el estrés hídrico y el conocimiento del punto de no retorno permite planificar las labores de siembra para garantizar 
su sobrevivencia en condiciones de campo. Se requieren nuevos estudios sobre el uso y distribución de los carbohidratos en la planta para comprender los aspectos de crecimiento y construcción de los tejidos que conforman la biomasa, además del análisis de embolismo y pérdida capacidad de absorción radicular con el fin de entender el impacto del estrés desde un ámbito hidráulico.

\section{CONFLICTOS DE INTERÉS}

Los autores declaran no tener conflicto de intereses.

\section{CONTRIBUCIÓN POR AUTOR}

Ambos autores son los únicos responsables de la obra en todos los aspectos que condujeron a la elaboración de su publicación.

\section{REFERENCIAS}

Blum, A. (2011). Plant Breeding for Water-Limited Environments. Amsterdam: Springer Science+Business Media.

https://doi.org/10.1007/978-1-4419-7491-4_2

Camareroa, J., Sánchez-Salguero, R., Sangüesa-Barredaa, G., Matías, L. (2018). Tree species from contrasting hydrological niches show divergent growthand water-use efficiency. Dendrochronologia, 52, 87-95.

https://doi.org/10.1016/j.dendro.2018.10.003

Caplan, J., Galanti, R., Olshevski, S. y Eisenman., R. (2019). Water relations of street trees in green infrastructure tree trench systems. Urban Forestry \& Urban Greening, 41, 170-178.

de la Rosa, J. M., Conesa, M. R., Domingo, R. y Pérez-Pastor, A. (2014). A new approach to ascertain the sensitivity to water stress of different plant water indicators in extra-early nectarine trees. Scientia Horticulturae, 169,147-153.

https://doi.org/10.1016/j.scienta.2014.02.021
Drechsler, K., Kisekkaa, I. y Upadhyaya, S. (2019). A comprehensive stress indicator for evaluating plant water status in almondtrees. Agricultural Water Management, 216, 214-223.

di Vaio, C., Marallo, N., Marino, G. y Caruso, T. (2013). Effect of water stress on dry matter accumulation and partitioning inpot-grown olive trees (cv Leccino and Racioppella). Scientia Horticulturae, 164(2013), 155-159.

https://doi.org/10.1016/j.scienta.2013.09.008

Ehrenberger, W., Rüger, S., Fitzke, R., Vollenweider, P., Günthardt-Goerg, P., Kuster, T., Zimmermann, U. y Arend, M. (2012). Concomitant dendrometer and leaf patch pressure probe measurements reveal the effect of microclimate and soil moisture on diurnal stem water and leaf turgor variations in young oak trees. Londres: Functional Plant Biology.

https://doi.org/10.1071/FP11206

Fernández, J. E., Rodriguez-Dominguez, C. M., Perez-Martin, A., Zimmermann, U., Rüger, S., Martín-Palomo, M. J., Torres-Ruiz, J. M., Cuevas, M. V., Sann, C., Ehren-berger, W. y Diaz-Espejo, A., (2011). Online-monitoring of tree water stress in a hedgerow olive orchard using the leaf patch clamp pressure probe. Agricultural Water Management, 100, 25-35.

Girón, I. F., Corell, M., Galindo, A., Torrecillas, E., Morales, D., Dell'Amico, J., Torrecillas, A., Moreno, F. y Moriana, A. (2015). Changes in the physiological response between leaves and fruits during a moderate water stress in table olive trees. Agricultural Water Management, 148, 280-286.

https://doi.org/10.1016/j.agwat.2014.10.024

Guerfel, M., Baccouri, O., Boujnah, D., Chaïbi, W. y Zarrouk, M. (2009). Impacts of water stress on gas exchange, water relations, chlorophyll content and leaf structure in the two main Tunisian olive (Olea europaea L.) cultivars. Scientia Horticulturae, 119, 257-263.

https://doi.org/10.1016/j.scienta.2008.08.006

Hammani, S. B. M., Costagli, G. y Rapoport, H. F. (2013). Cell and tissue of olive endo-carp sclerication vary according to water availability. Physiologia Plantarum, 149, 571-582. 
Instituto Meteorológico Nacional (IMN) (2018). Condiciones meteorológico nacionales. Recuperado de

http://www.imn.ac.cr

Krause, G. H., Winter, K., Matsubara, S., Krause, B., Jahns, P., Virgo, A., Aranda, J. y García, M. (2012). Photosynthesis, photoprotection, and growth of shade-tolerant tropical tree seedlings under full sunlight. Photosynthesis Research, 113, 237-285.

https://doi.org/10.1007/s11120-012-9731-z

Lim, T. K. (2013). Edible Medicinal and Non-Medicinal Plants. Amsterdam: Springer Netherlands.

https://doi.org/10.1007/978-94-007-7395-0_64

López-López, M., Espadafor, M., Testia, L., Loriteb, I., Orgaza, F., Fereres, E. (2018). Water use of irrigated almond trees when subjected to water deficits. $\mathrm{Ag}$ ricultural Water Management, 195, 84-93.

Maatallah, M., Ghanem, M. E., Albouchi, A., Bizid, E. y Lutts, S. (2010). A greenhouse investigation of responses to different water stress regimes of Laurus nobilis trees from two climatic regions. Journal of Arid Environments, 74, 327-337.

https://doi.org/10.1016/j.jaridenv.2009.09.008

Myers, B. J. (1988). Water stress integral a link between short term stress and long term growth. Tree Physiology, 4, 315-323.

Ortuño, F. M., García-Orellana, T., Conejero, W., Ruiz-Sánchez, C. M., Alarcón, J. y Torrecillas, A. (2006). Stem and leaf water potentials, gas exchange, sap flow, and trunk diameter fluctuations for detecting water stress in lemon trees. Trees, 20, 1-8.

https://doi.org/10.1007/s00468-005-0004-8

Pedrero, F., Maestre-Valero, J. F., Mounzer, O., Alarcón, J. J. y Nicolás, E. (2014). Physiological and agronomic mandarin trees performance under saline reclaimed water combined with regulated deficit irrigation. Agricultural Water Management, 146, 228-237.

https://doi.org/10.1016/j.agwat.2014.08.013

Regent Instrument (2012). WinFOLIA pro 2012. Boston: Regent Instrument Inc. Recuperado de https:// www.regentinstruments.com

Rodriguez-Dominguéz, C. M., Ehrenberger, W., Sann, S., Rüger, S., Sukhorukov, V., Martín-Palomo, M. J., Diaz-Espejo, A., Cuevas, M. V., Torres-Ruiz, J. M.,
Perez-Martin, A., Zimmermann, U. y Fernández, J. E. (2012). Concomitant measurements of stem sap flow and leaf turgor pressure in olive trees using the leaf patch clamp pressure probe. Agricultural Water Management, 114, 50-58.

https://doi.org/10.1016/j.agwat.2012.07.007

Roussos, P. A., Denaxa, N. K., Damvakaris, T., Stournaras, V. y Argyrokastritis, I. (2010). Effect of alleviating products with different mode of action on physiology and yield of olive under drought. Scientia Horticulturae, 125, 700-711.

https://doi.org/10.1016/j.scienta.2010.06.003

Sanchez-Costa, E., Poyatos, R. y Sabate, S. (2015). Contrasting growth and water use strategies in four co-occurring Mediterranean tree species revealed by concurrent measurements of sap flow and stem diameter variations. Agricultural and Forest Meteorology, 207, 24-37.

https://doi.org/10.1016/j.agrformet.2015.03.012

Statsoft (2015). Statistica, version 9.0. Londres: Statsoft. Recuperado de http://www.statsoft.com

Szota, C., Coutts, A., Thom, J., Virahsawmy, H., Fletcher, T. y Livesley, S. (2019). Street tree stormwater control measures can reducer un off but may not benefit established trees. Landscape and Urban Planning, 182, 144-155.

Tong, X., Mu, Y., Zhang, J., Meng, P. y Li, J. (2019). Water stress controls on carbon flux and water use efficiency in a warm-temperate mixed plantation. Journal of Hydrology, 571, 669-678.

Varone, L., Ribas-Carbo, M., Cardona, C., Gallé, A., Medrano, H., Gratani, J. y Flexas, J. (2012). Stomatal and non-stomatal limitations to photosynthesis in seedlings and saplings of Mediterranean species pre-conditioned and aged in nurseries: Different response to water stress. Environmental and Experimental Botany, 75, 235-247.

https://doi.org/10.1016/j.envexpbot.2011.07.007

Westhoff, M., Zimmermann, D., Schneider, H., Wegner, L. H., Geßner, P., Jakob, P., Bamberg, E., Shirley, S., Bentrup, F. W. y Zimmermann, U. (2009). Evidence for discontinuous water columns in the xylem conduit of tall birch trees. Plant Biology, 11, 307-327. 
Zaharah, A. R., Bah, A. R., Mwange, N. X., Kathuli, P. y Juma, P. (1999). Management of Gliricidia (Cliricidia sepium) residues for improved sweet corn yield in an ultisol. Nutrient Cycling in Agroecosystems, 54, 31-39.

Zimmermann, U., Schneider, H., Wegner, L. H. y Haase, A. (2004). Water ascent in tall trees: does evolution of land plants rely on a highly metastable state? New Phytologist (Tansley Review), 162, 575-615.

Zimmermann, D., Westhoff, M. y Zimmermann, G. (2007). Foliar water supply of tall trees: evidence for mucilage-facilitated moisture uptake from the atmosphere and the impact on pressure bomb measurements. Protoplasma, 232, 11-34.

Zimmermann, D., Reuss, R., Westhoff, M., Geßner, P., Bauer, W., Bamberg, E., Bentrup, F. W. y Zimmermann, U. (2008). A novel, non-invasive, online-monitoring, versatile and easy plant-based probe for measuring leaf water status. Journal of Experimental Botany, 59(11), 3157-3167.

https://doi.org/10.1093/jxb/ern171

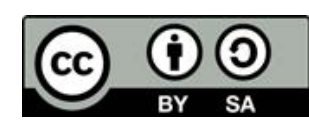

Colombia Forestal •ISSN 0120-0739 • e-ISSN 2256-201X • Bogotá-Colombia • Vol. 23 No. 1 • Enero-Junio de 2020 • pp. 20-34 